\title{
Accuracy of Coracoid Bone Graft Placement: Open versus Arthroscopic Latarjet
}

\author{
Adriano Russo ${ }^{1}$ Andrea Grasso ${ }^{2}$ Annalisa Arrighi ${ }^{3}$ \\ ${ }^{1}$ Department of Orthopaedics, Florence Institute of Assistance and \\ Care (IFCA) Ulivella and Glicini Clinic, Florence, Italy \\ 2 Department of Orthopaedics, Villa Valeria Clinic, Rome, Italy \\ ${ }^{3}$ School of Medical and Pharmaceutical Sciences, University of Genoa, \\ Genoa, Italy \\ ${ }^{4}$ Department of Epidemiology and Biostatistics, Giannina Gaslini \\ Institute, Genoa, Italy \\ ${ }^{5}$ Research Center of Osteoporosis and Osteoarticular Diseases, \\ University of Genoa, Genoa, Italy
}

Joints 2017;5:85-88.

\section{Angela Pistorio $^{4} \quad$ Luigi Molfetta ${ }^{5}$}

\begin{abstract}
Address for correspondence Adriano Russo, MD, Orthopaedic Florence Institute (IFCA), Via Fieschi N. 400, 19131 La Spezia, Italy (e-mail: studiomedico.russo@libero.it).
\end{abstract}

\begin{abstract}
Keywords

- arthroscopic

- coracoid graft

- instability

- Latarjet-Patte

- shoulder

- computed tomography

Purpose The aim of this study was to compare the accuracy of the coracoid bone graft placement with the open Latarjet-Patte and arthroscopic Latarjet (arthro-Latarjet) procedures in the treatment of anterior instability of the shoulder.

Methods Forty-six patients affected by anterior shoulder instability were divided into two groups. In group A $(n=25)$, patients were operated by arthroscopic Latarjet (arthro-Latarjet) procedure and in group B $(n=21)$, patients were operated by open Latarjet-Patte procedure. Instrumental investigation was based on three-dimensional computed tomography (3D-CT) at a minimum 1-year follow-up. Graft placement and integration, divergence and posterior protrusion of the screws, and glenohumeral osteoarthritis were considered as outcomes. Statistical analysis was performed with chi-square or Fisher's exact test. Significance was set at $p<0.05$.

Results Positioning of the coracoid graft proved to be optimal in 76\% (19/25) of patients of group A and in 100\% (21/21) of patients of group B (Fisher's exact test, $p=0.025)$. Screw placement with respect to the glenoid surface showed a posterior divergence in $44 \%(11 / 25)$ of patients in group $A$ and in $24 \%(5 / 21)$ of patients in group $B(p=0.15)$. Posterior protrusion of screw was observed in $76 \%(19 / 25)$ of patients in group $A$ and $71.4 \%(15 / 21)$ of patients in group B $(p=0.73)$. Graft integration was present in $76 \%(19 / 25)$ of patients in group $A$ and $85.7 \%(18 / 21)$ of patients in group $B$ (Fisher's exact test, $p=0.48$ ). Mild signs of glenohumeral osteoarthritis were observed in $12 \%(3 / 25)$ of patients in group A and $28.6 \%(6 / 21)$ of patients in group B (Fisher's exact test, $p=0.26$ ).

Conclusion Patients operated with open Latarjet-Patte procedure showed better results than those of the arthro-Latarjet group in reference to the positioning of the graft on the coronal plane $(p=0.025)$. No significant differences between the groups were observed for graft integration, divergence of the screws, posterior protrusion of the screws, and osteoarthritis.

Level of Evidence Level II, nonrandomized prospective comparative study.
\end{abstract}

DOI https://doi.org/ $10.1055 / \mathrm{s}-0037-1603934$. ISSN 2282-4324.
Copyright @ 2017 Georg Thieme Verlag KG Stuttgart · New York 


\section{Introduction}

The Latarjet technique has proven over the years to be a very effective and reliable procedure for the surgical treatment of anterior instability of the shoulder, especially in cases of glenoid bone loss, bipolar (humeral head and anterior glenoid) bone defects, and revision of surgical failures. ${ }^{1-4}$ Also, the introduction of subscapularis muscle split, described by Patte, improved the results concerning recovery of external rotation. ${ }^{5}$ In 2007, Lafosse et al described the arthroscopic Latarjet procedure (arthro-Latarjet) as an alternative to traditional open surgical technique with encouraging short-term results. ${ }^{6}$ The proper placement of the coracoid graft on the anterior glenoid neck represents the most important step of the Latarjet procedure for the restoration of shoulder stability and maintenance of results over time. ${ }^{7}$ However, no clear information exist about efficacy of open and arthroscopic Latarjet procedure in terms of correct coracoid graft placement.

The purpose of this study was to assess the accuracy of coracoid graft placement in the open and arthroscopic Latarjet procedure for the treatment of anterior shoulder instability. The hypothesis of the study was that the difference between the two technique is not significant.

\section{Methods}

Forty-six patients (43 males and 3 females) were prospectively and consecutively enrolled for this study and divided into two groups: 25 patients were operated with arthroscopic "arthro-Latarjet" technique (group A) and 21 patients underwent an open Latarjet-Patte procedure (group B). All of them were suffering from anterior instability of the shoulder and had an instability severity index score (ISIS) equal to or greater than 6 points. ${ }^{8,9}$ Exclusion criteria were: age older than 50 years, concomitant rotator cuff tears, multidirectional instability, and systemic disorders such as autoimmune or rheumatic diseases.

Arthroscopic technique was performed with five portals: A-D-E-H-M. The open technique was performed through the deltopectoral approach. The following steps were followed in both the procedures: coracoid preparation and osteotomy, subscapularis muscle split, preparation of the anterior glenoid neck, and fixation of the coracoid graft to the anterior side of the glenoid with two 3.5-mm cannulated titanium screws of length varying between 36 and $38 \mathrm{~mm}$. In both the groups, we used the arthro-Latarjet instruments (Mitek, Johnson \& Johnson, New Brunswick, New Jersey, United States) for coracoid graft placement. The same surgeon (A.R.) operated all the patients.

At a minimum 1-year follow-up, all the patients underwent an imaging evaluation with standard anteroposterior axillary radiographs and a three-dimensional computed tomography (3D-CT). The following parameters were examined: positioning of the coracoid graft on the sagittal and coronal plane, placement of the screws with respect to the glenoid surface, screw length with respect to the anteroposterior width of the glenoid neck, integration of the bone graft, and signs of osteoarthritis. ${ }^{10}$

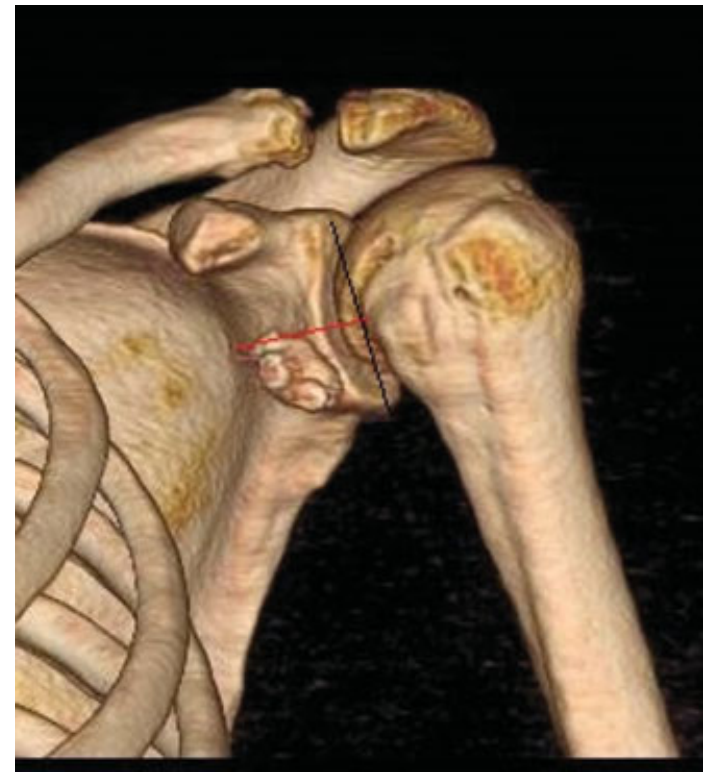

Fig. 1 Position of the graft compared with equator of the glenoid (three-dimensional computed tomography [3D-CT] coronal scan).

Positioning of the coracoid graft on the coronal plane was classified as "adequate" if the graft was placed in a subequatorial position or "imperfect" if the graft was placed at the level or over the equator ( - Fig. 1). Positioning of the coracoid graft on the axial plane was classified as "adequate" if the graft was flat compared with the glenoid surface or moved within 2 to $5 \mathrm{~mm}$ medially (flush graft) or "imperfect" if hanging laterally more than $2 \mathrm{~mm}$ compared with the anterior glenoid edge (proud graft) or shifted medially more than $5 \mathrm{~mm}$ compared with the glenoid edge (recessed graft), (-Fig. 2 ).

Screw positioning was assessed on axial CT scans. Screw divergence was measured as the angle that the screw formed with the articular glenoid surface and was open anteriorly

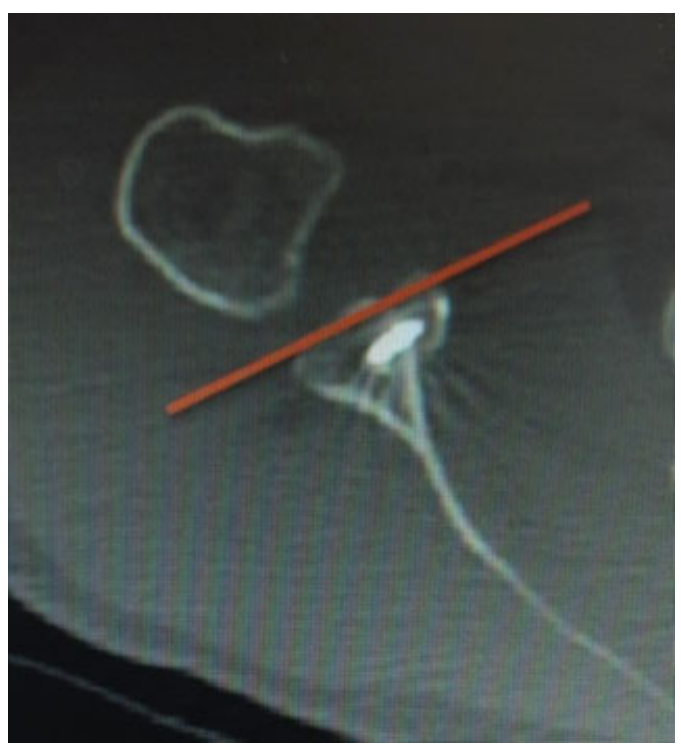

Fig. 2 Position of the graft compared with anterior glenoid edge (computed tomography [CT] axial scan). 


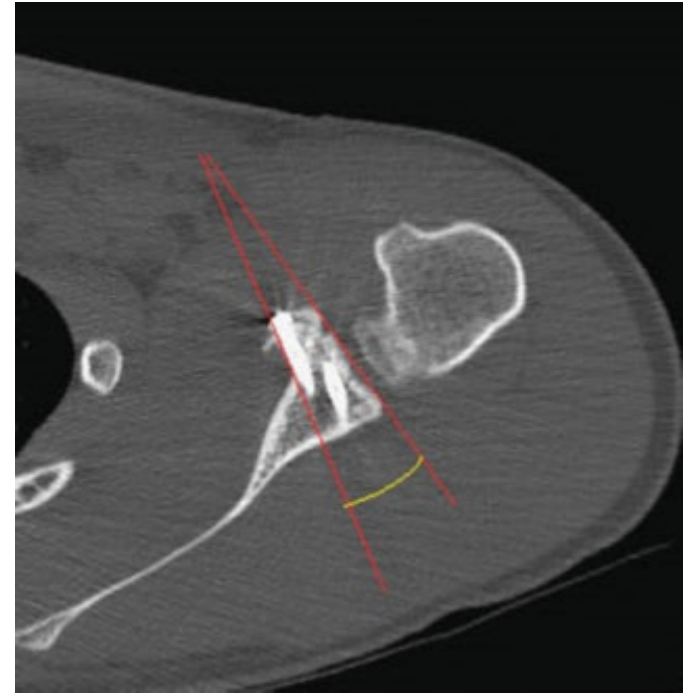

Fig. 3 Divergence angle is formed by the screw direction and the tangent to the glenoid surface (computed tomography [CT] axial scan).

(convergence angle: the tip of the screw points toward the posterior edge of the articular surface) or posteriorly (divergence angle: the tip of the screw diverges from the posterior edge of the articular surface, - Fig. 3). The divergence angle of the screws should be within 10 to 15 degrees to avoid injuries to the suprascapular nerve. ${ }^{11}$

Screw length was assessed for screw tip protrusion from the posterior glenoid neck and measured in millimeters (-Fig. 4).

Graft integration was defined as the disappearance in the axial CT scans of a separation between the coracoid graft and the anteroinferior glenoid bone with inability to identify the two contiguous cortical profiles. Integration was semiquantitatively rated as partial (limited to a portion varying from 35 to $75 \%$ of the contact area between the graft and the

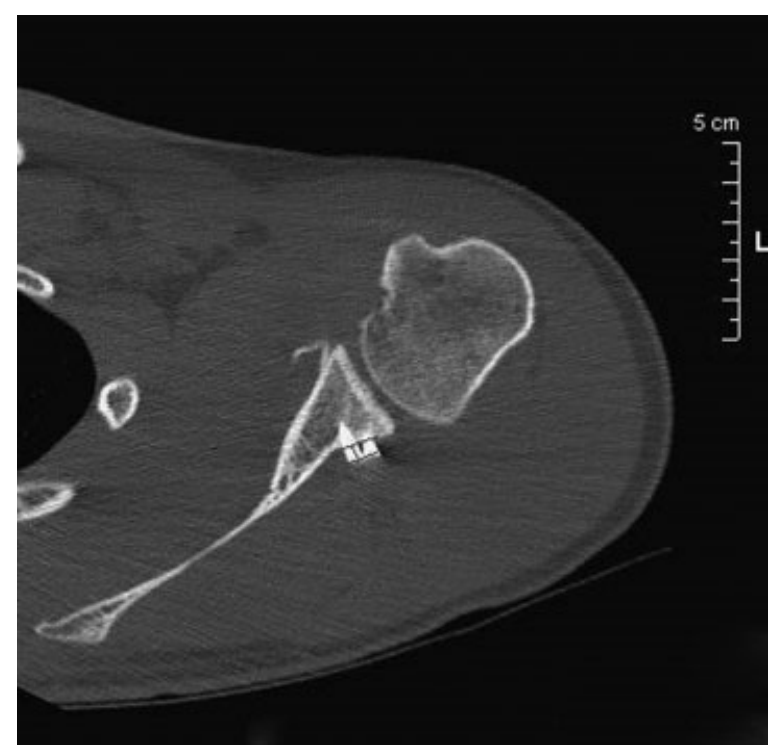

Fig. 4 Measurement of the posterior protrusion of the screw (computed tomography [CT] axial scan). glenoid surface) or complete (if greater than 75\%). Nonunion was defined as persistence of two distinct cortical profiles (graft and glenoid neck) and/or fragmentation of the graft.

Glenohumeral osteoarthritis was assessed according to Samilson and Prieto grading system. ${ }^{12}$

Statistical analysis of data was performed with the MannWhitney $U$ test for continuous variables and Fisher's exact test for categorical variables. Significance was set at $p<0.05$.

\section{Results}

In group A, on axial CT scans, the graft was "flush" in 24 cases (96\%) and "proud" in 1 (4\%); no recessed grafts were found. On sagittal CT scans, graft placement was adequate (subequatorial) in 20 cases (80\%) and imperfect (equatorial) in 5 (20\%). Overall, positioning of the graft was correct (flush and subequatorial) in 19 cases (76\%). No nonunions were found. In group B, the graft was "flush" and subequatorial (correct positioning) in all cases. Comparison between the groups showed a significant difference for overall coracoid graft positioning on the coronal plane ( $p=0.025)$.

Graft integration in group A was complete in 19 cases (76\%) and partial in 6 (24\%). Graft integration in group B was complete in 18 cases ( $86 \%$ ) and partial in $3(14 \%)$. No significant difference between the groups was observed ( $p=0.39$ ).

Assessment of screw placement showed that in group A, screws were parallel to the glenoid surface in 14 cases (56\%) and divergent in 11 (44\%). Median divergence angle was 19.4 degrees (range, 10-32 degrees). In group B, screws were parallel to the glenoid surface in 16 (76\%) and divergent in 5 (24\%) cases. Median divergence angle was 11.2 degrees (range, 6-18 degrees). No significant difference between the groups was observed for screw divergence $(p=0.15)$. Posterior protrusion of screw was absent in 4 (16\%), <2 mm in 18 (72\%), and $\geq 2 \mathrm{~mm}$ in $3(12 \%)$ cases. Median screw protrusion was $1.8 \mathrm{~mm}$ (range, $0-3 \mathrm{~mm}$ ). Screw posterior protrusion was observed in 76\% (19/25) of cases in group A and $71.4 \%(15 / 21)$ of cases in group B. Posterior protrusion of screws was $<2 \mathrm{~mm}$ in 16 cases (76\%) and $\geq 2 \mathrm{~mm}$ in 5 cases (24\%). Median screw protrusion was $1.8 \mathrm{~mm}$ (range, 0-3 mm). The difference between the groups for posterior protrusion of screw was not significant $(p=0.73)$.

Assessment of glenohumeral osteoarthritis (OA) according to Samilson and Prieto classification showed that in group A, OA was absent in $22(88 \%)$ cases, grade 1 in 2 ( $8 \%$ ) cases, and grade 2 in 1 (4\%) case. In group B, OA was absent in 15 (71\%) cases, grade 1 in 5 (24\%) cases, and grade 2 in 1 (5\%) case. No significant difference between the groups was observed for glenohumeral OA ( $p=0.26$ ).

Mild signs of glenohumeral OA were observed in $12 \%$ of cases (3/25) in group A and 28.6\% (6/21) in group B (Fisher's exact test, $p=0.26$ ).

\section{Discussion}

Many surgical techniques have been described for the treatment of anterior instability of the shoulder. The goal of any surgical treatment should be to stabilize the shoulder 
without compromising strength or range of motion. In 1954 , Latarjet described his treatment for recurrent dislocation of shoulder by transposing the coracoid process on the neck of the scapula and securing it with a screw. ${ }^{13}$ The method underwent several changes over the years, but the basic procedure remained unchanged for coracoid osteotomy and fixation to the anterior glenoid neck. The critical step of the procedure is the proper placement of the coracoid graft to the anterior glenoid, where it is generally fixed with two $3.5-\mathrm{mm}$ screws, 36 to $38 \mathrm{~mm}$ in length. This size is recommended to prevent nonunion. ${ }^{14}$ The efficacy of double screw fixation is mainly related to an improved contact surface and rotational stability of the graft. ${ }^{15}$

Many authors investigated the bone block position on radiographs. Allain et $\mathrm{al}^{16}$ observed $53 \%$ too lateral bone blocks (proud grafts) and 5\% too medial bone blocks (recessed grafts). Cassagnaud et $\mathrm{al}^{17}$ reported more than $10 \%$ of bone blocks protruding into the joint on CT scans. Hovelius et $\mathrm{al}^{18}$ found $36 \%$ of bone blocks seated above the equator and $6 \%$ bone blocks placed too medially. Huguet et $\mathrm{al}^{19}$ found $45 \%$ of the grafts protruding into the joint. ${ }^{19}$ All these studies demonstrated the importance of the position of the graft, which is directly related to the outcome. A graft placed too lateral or protruding into the joint raises OA over time, while a coracoid graft placed too medially and/or above the equator can generate recurring instability.

Nevertheless, optimal position of the coracoid process grafts is difficult to define; in general, it is believed that it should be below the equator, not too medial from the anterior glenoid edge, and less than 2 to $10 \mathrm{~mm}$ from the cartilage according to different authors. ${ }^{20,21}$ In our study, according to previous reports, we used a careful 3D-CT scan assessment of some imaging parameters useful to evaluate differences between open and arthroscopic Latarjet regarding graft placement, fixation, and healing, and found that open procedure provided better results in terms of positioning of the graft on the coronal plane. With regard to other parameters, graft integration, posterior screw divergence, posterior protrusion of the screws, and osteoarthritis, data analysis showed no significant differences between the groups.

\section{References}

1 van der Linde JA, van Wijngaarden R, Somford MP, van Deurzen DF, van den Bekerom MP. The Bristow-Latarjet procedure, a historical note on a technique in comeback. Knee Surg Sports Traumatol Arthrosc 2016;24(02):470-478

2 Emami MJ, Solooki S, Meshksari Z, Vosoughi AR. The effect of open Bristow-Latarjet procedure for anterior shoulder instability: a 10-year study. Musculoskelet Surg 2011;95(03):231-235

3 Hovelius L, Vikerfors O, Olofsson A, Svensson O, Rahme H. Bristow-Latarjet and Bankart: a comparative study of shoulder stabilization in 185 shoulders during a seventeen-year follow-up. J Shoulder Elbow Surg 2011;20(07):1095-1101
4 Provencher MT, Bhatia S, Ghodadra NS, et al. Recurrent shoulder instability: current concepts for evaluation and management of glenoid bone loss. J Bone Joint Surg Am 2010;92(Suppl 2): 133-151

5 Joshi MA, Young AA, Balestro JC, Walch G. The Latarjet-Patte procedure for recurrent anterior shoulder instability in contact athletes. Orthop Clin North Am 2015;46(01):105-111

6 Lafosse L, Lejeune E, Bouchard A, Kakuda C, Gobezie R, Kochhar T. The arthroscopic Latarjet procedure for the treatment of anterior shoulder instability. Arthroscopy 2007;23(11):1242.e1-1242.e5

7 Nourissat G, Delaroche C, Bouillet B, Doursounian L, Aim F. Optimization of bone-block positioning in the Bristow-Latarjet procedure: a biomechanical study. Orthop Traumatol Surg Res 2014;100(05):509-513

8 Balg F, Boileau P. The instability severity index score. a simple preoperative score to select patients for arthroscopic or open shoulder stabilisation. J Bone Joint Surg Br 2007;89(11):1470-1477

9 Bouliane M, Saliken D, Beaupre LA, Silveira A, Saraswat MK, Sheps DM. Evaluation of the instability severity index score and the Western Ontario Shoulder Instability Index as predictors of failure following arthroscopic Bankart repair. Bone Joint J 2014;96-B (12):1688-1692

10 Gordins V, Hovelius L, Sandström B, Rahme H, Bergström U. Risk of arthropathy after the Bristow-Latarjet repair: a radiologic and clinical thirty-three to thirty-five years of follow-up of thirty-one shoulders. J Shoulder Elbow Surg 2015;24(05):691-699

11 Lädermann A, Denard PJ, Burkhart SS. Injury of the suprascapular nerve during Latarjet procedure: an anatomic study. Arthroscopy 2012;28(03):316-321

12 Samilson RL, Prieto V. Dislocation arthropathy of the shoulder. J Bone Joint Surg Am 1983;65(04):456-460

13 Latarjet M. Technic of coracoid preglenoid arthroereisis in the treatment of recurrent dislocation of the shoulder. Lyon Chir 1958;54(04):604-607

14 Pichon H, Startun V, Barthelemy R, Saragaglia D. Comparative study of the anatomic and clinical effect of Weaver or subtotal subscapularis tendon section in Latarjet procedure [Article in French]. Rev Chir Orthop Repar Appar Mot 2008;94(01):12-18

15 Di Giacomo G, Costantini A, de Gasperis N, et al. Coracoid graft osteolysis after the Latarjet procedure for anteroinferior shoulder instability: a computed tomography scan study of twenty-six patients. J Shoulder Elbow Surg 2011;20(06):989-995

16 Allain J, Goutallier D, Glorion C. Long-term results of the Latarjet procedure for the treatment of anterior instability of the shoulder. J Bone Joint Surg Am 1998;80(06):841-852

17 Cassagnaud X, Maynou C, Mestdagh H. Results of 106 Latarjet-Patte procedures: computed tomography analysis at 7.5 years follow-up. Rev Chir Orthop Repar Appar Mot 2003;89(08):683-692

18 Hovelius L, Körner L, Lundberg B, et al. The coracoid transfer for recurrent dislocation of the shoulder. Technical aspects of the Bristow-Latarjet procedure. J Bone Joint Surg Am 1983;65(07): 926-934

19 Huguet D, Pietu G, Bresson C, Potaux F, Letenneur J. Anterior instability of the shoulder in athletes: apropos of 51 cases of stabilization using the Latarjet-Patte intervention. Acta Orthop Belg 1996;62(04):200-206

20 Walch G, Charret P, Pietro-Paoli H, Dejour H. Anterior recurrent luxation of the shoulder. Postoperative recurrences. Rev Chir Orthop Repar Appar Mot 1986;72(08):541-555

21 Kraus TM, Graveleau N, Bohu Y, Pansard E, Klouche S, Hardy P. Coracoid graft positioning in the Latarjet procedure. Knee Surg Sports Traumatol Arthrosc 2016;24(02):496-501 